\title{
The musculo-skeletal features of hypothyroidism
}

\author{
Douglas N. Golding \\ M.D., F.R.C.P.I.
}

\section{Consultant in Rheumatology, Princess Alexandra Hospital and Harlow Group}

Summary

Patients with hypothyroidism may present in rheumatism departments with muscle pain and weakness, acroparaesthesiae or less commonly definite myopathy. Osteolytic lesions are rare and require further study.

DURING recent years it has become accepted that patients with hypothyroidism may present with a variety of rheumatic syndromes, sometimes well before the more obvious features of myxoedema have appeared, and these 'hypothyroid-rheumatic syndromes' will be described. This term is used in preference to 'myxoedematous arthropathy', because the patients in question are not frankly myxoedematous, myalgia is more frequent than arthralgia, and joint destruction is only an occasional feature.

The various musculo-skeletal syndromes associated with hypothyroidism are of clinical interest because of the vast number of rheumatic conditions that may be simulated. For example, either osteoarthritis of peripheral joints or the spine, rheumatoid arthritis, atypical gout, or non-articular rheumatism may be initially suspected. However, since the above conditions occur much more frequently than the hypothyroid-rheumatic syndromes, every effort must be made to exclude them by clinical, radiological and laboratory investigations. Also, it must be remembered that osteoarthritis is very common in this age group, and that clinical gout may occur as a result of hyperuricaemia which may be associated with myxoedema, and consequently these conditions themselves can give rise to symptoms in addition to those features due to hypothyroidism.

The 'hypothyroid-rheumatic syndromes' which have been documented are listed in Table 1. Mention must also be made of auto-immune thyroiditis (Hashimoto's disease) as a possible cause of rheumatic pain. It seems possible that aching and stiffness, particularly affecting the neck muscles, trapezii and upper dorsal muscles may be a feature of active auto-immune thyroiditis in the premyxoedematous stage. This has been commented
TABLE 1. Hypothyroid-rheumatic syndromes

(1) Muscle pain and stiffness (Golding, 1970)

(3) Arthralgia, synovial thickening with effusion (Bland \& Frymoyer, 1970)

(3) Osteolytic lesions (Bland \& Frymoyer, 1970)

(4) Hypothyroid myopathy

(5) Acroparaesthesiae

upon by Doniach (personal communication). Becker et al. (1963), in a retrospective study of 506 patients with Hashimoto's thyroiditis, found that forty had 'secondary fibrositis' not associated with myxoedema - joint or muscle stiffness and aching, often maximal on rising in the morning-and sixty had thoracic pain. Although it is difficult to exclude osteoarthritis in such a retrospective series of patients, these figures are suggestive of an association between auto-immune thyroiditis and rheumatism, and the symptoms described are very reminiscent of those in hypothyroid patients presenting with muscle pain and stiffness.

Hypothyroidism presenting with muscle pain and stiffness

Golding (1970) described nine hypothyroid patients presenting with muscle and joint pains and stiffness: the various clinical features and their frequency is given in Table 2. Hypothyroidism was suspected on account of lethargy, cold-sensitivity, weight-gain or mental dullness, but only one patient looked typically myxoedematous. Notable features were: aggravation of pain and stiffness by cold weather (as might be expected in hypothyroid patients, who often complain of cold-sensitivity); aggravation by phenylbutazone (which has an antithyroid action); and stiffness most pronounced in the early morning, sometimes lasting for $30 \mathrm{~min}$ or more and initially raising suspicion of rheumatoid arthritis or polymyalgia rheumatica. Muscle cramps (usually affecting the calves) occurred in most patients. Five had acroparaesthesiae, and carpal tunnel syndromes were established by median nerve 
TABle 2. Features in nine hypothyroid patients presenting with muscle pain and stiffness (from Golding, 1970)

\begin{tabular}{lc}
\hline & $\begin{array}{c}\text { No. of } \\
\text { patients }\end{array}$ \\
\hline Muscle pain & 9 \\
Muscle stiffness & 8 \\
Cramps & 6 \\
Acroparaesthesiae & 5 \\
\hline
\end{tabular}

conduction tests in three. There was a universally good response to thyroid, with parallel improvement in the electrocardiogram voltage and $\mathrm{T}$-wave disposition.

Pain and stiffness also occurred in the majority of patients in Bland \& Frymoyer's series (1970) (Table 3), and here also there was a complete symptomatic response to thyroid administration.

\section{Hypothyroidism with arthralgia, synovial thickening and effusion}

In a series of thirty-eight patients with proven hypothyroidism, thirteen had joint pain and synovial thickening affecting principally knees and small joints of the hands and feet, often with synovial effusions (Bland \& Frymoyer, 1970; Table 3). Although in the past these features have been sporadically noted in myxoedematous patients, this is the first report of a definite arthropathy occurring in association with hypothyroidism. The synovial thickening was not due to inflammatory synovitis, and the appearance and composition of the synovial fluid was normal. It is postulated that the condition is due to hyaluronic acid deposition in the connective tissue, ligaments and other joint structures leading to laxity of ligaments, joint instability, and consequent synovial effusion. Hyperuricaemia can occur in myxoedema (Leeper et al., 1960), and in this series four patients had a serum uric acid over $7 \mathrm{mg} / 100 \mathrm{ml}$, so it is possible that in this group the symptoms may have been at least partially due to secondary gout. However, if this were the case one would expect to have found lowviscosity synovial fluid with a high leucocyte count and, in particular, a high concentration of intra- and extra-cellular urate crystals in the fluid. Synovial thickening was not observed in any of Golding's series, and this would seem to be a distinct entity occurring in a proportion of patients with hypothroidism. As already mentioned, all Bland \& Frymoyer's patients recovered on thyroid replacement therapy.

\section{Osteolytic lesions in hypothyroidism}

Thyroxine deficiency may result in characteristic bone changes if the deficiency occurs during the years of growth. There may be a characteristic X-ray appearance, sometimes termed epiphyseal dysgenesis, due to defective epiphyseal ossification (Wilkins, 1941). High levels of serum calcium and alkaline phosphatase, corrected by thyroid therapy, may occur in cretinism (Talbot, 1939). Wilkins (1957) described a 7-year-old girl with hypothyroidism who developed a painful epiphyseal lesion in one hip, with flattening and fragmentation of the femoral head. Weissbein et al. (1959) reported a young man with adult myxoedema who presented with hip-pain due to an osteolytic lesion in the femoral head, which improved clinically and radiologically with thyroid therapy. Bywaters (1969) described cystic lesions in the ends of the phalanges in myxoedema, considered to be due to defective development of bone from cartilage.

An interesting osteolytic lesion of perhaps similar nature occurred in three of Bland \& Frymoyer's patients (Fig. 1). There was compression and crumbling of the tibial plateau with marked loss of bone density, resembling the bone necrosis occasionally seen in severe cases of rheumatoid arthritis, especially after frequent intra-articular steroid injections. It would seem possible that this represents actual infiltration of bone with hyaluronic acid-like substance, and in future instances it would be of

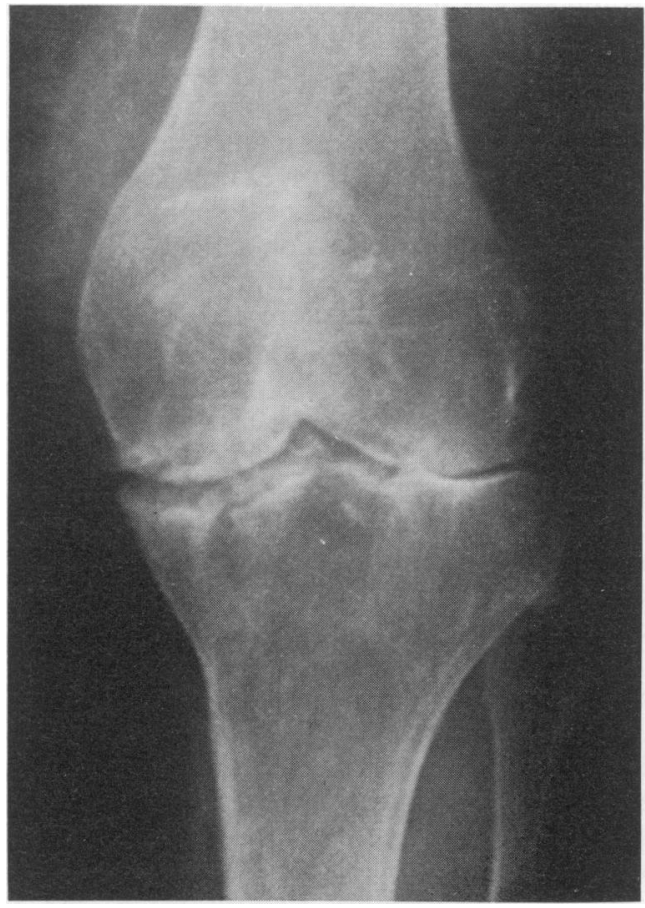

Fig. 1. Bone lesion (right knee-joint) in a case of hypothyroidism. (By kind permission of Dr J. H. Bland.) 


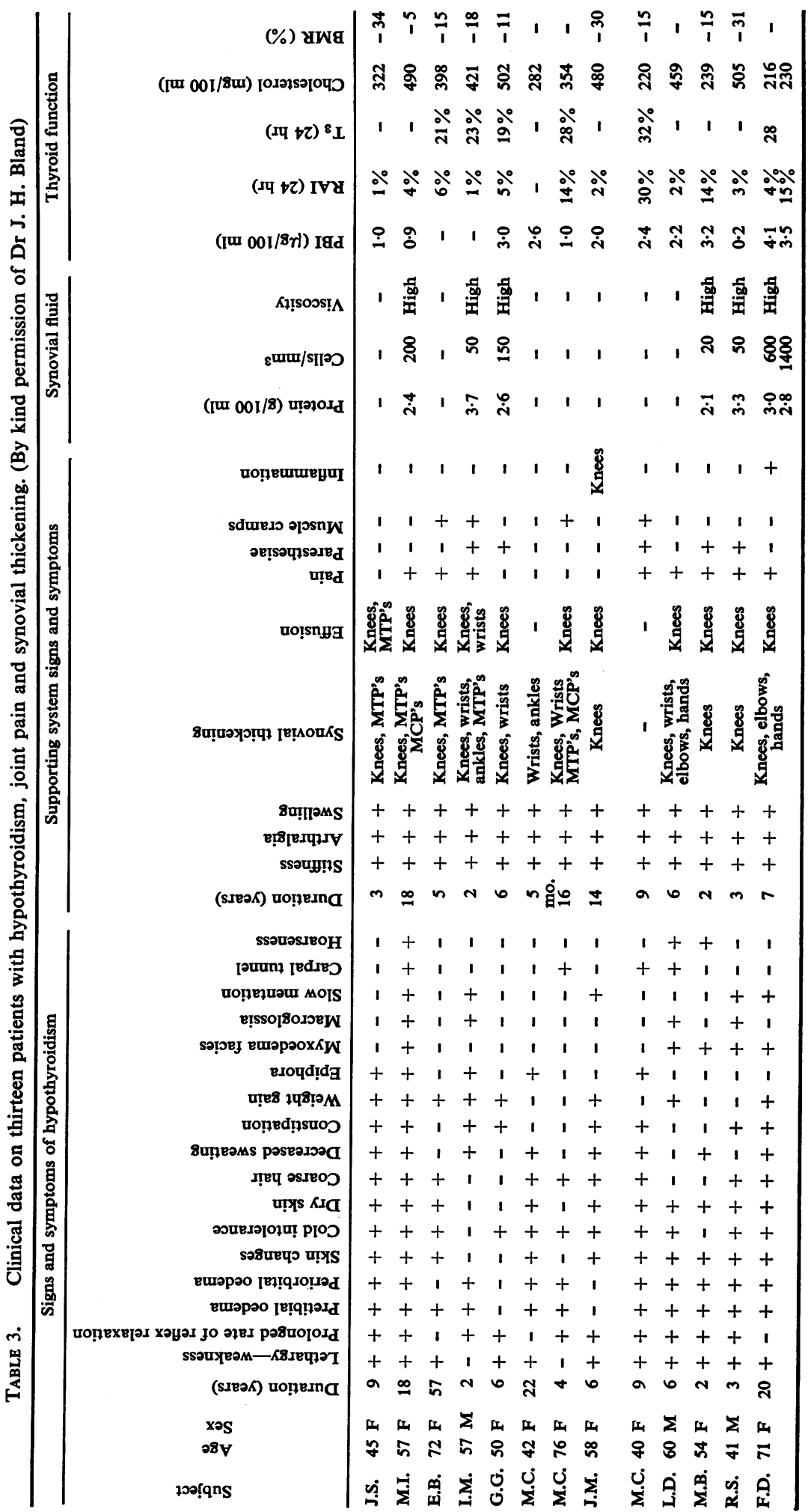


great interest to carry out bone biopsies, which as yet have never been attempted in these cases.

\section{Hypothyroid myopathy}

The majority of patients with myxoedema are weak, and it is possible that they have a minor degree of myopathy. The fact that the serum creatine-kinase and (less frequently) the SGOT and aldolase are raised in most cases of hypothyroidism (Griffiths, 1965) lends weight to this possibility. Only a small proportion of hypothyroid patients are affected with severe muscle weakness as the predominant feature. The weakness principally affects the proximal muscle groups, and in the absence of treatment spreads distally.

Hypotonic weakness with or without muscle atrophy is usually the case in severe hypothyroid myopathy, but myotonic, myasthenic and pseudohypertrophic forms very rarely occur. The pseudohypertrophic type has been described in acquired myxoedema (Hoffman's syndrome) and in congenital hypothyroidism (the Debré-Sémélaigne syndrome). The more usual form, hypotonic myopathy without hypertrophy, is exemplified by three cases described by Fessel (1968). The symptoms closely resembled polymyositis-marked weakness associated with muscle pain and increase in serum muscle enzymes. In two patients the muscular symptoms were not abolished after several months of thyroid therapy, illustrating an important difference between hypothyroid myopathy and the 'hypothyroidrheumatic syndromes' already described, where there is always a good response to thyroid. This probably reflects a different pathology underlying the two conditions-hyaluronic acid accumulation in the hypothyroid-rheumatic syndromes, intrinsic muscle lesions with fibre atrophy and interstitial histiocyte infiltration in hypothyroid myopathy. The latter, in effect, may be considered as a form of polymyositis secondary to hypothyroidism. Electron microscopy showed significant changes in muscle mitochondria by electron microscopy in a case of hypothyroid myopathy described by Norris \& Panner (1966). It is possible that thyroid deficiency can cause direct damage to muscle membranes and fibres. Electromyography has also shown changes suggestive of polymyositis in patients with this condition (Salick et al., 1968).

\section{Acroparaesthesiae}

Finally, hypothyroid patients may present with paraesthesiae of the hands and feet. This is usually a feature of median or medial popliteal nerve compression in the carpal or tarsal tunnels, but it should be borne in mind that in some very advanced cases of myxoedema deposition of pseudo-mucinous substance (presumably hyaluronate) has been observed in tendon and nerve sheaths; and also that an intrinsic hypothyroid neuropathy, in which there is (predominantly sensory) nerve conduction slowing, diminished deep reflexes, paraesthesiae and slight distal impairment of sensation, has been demonstrated in some cases (Fincham \& Cape, 1968).

\section{References}

Becker, K. L., Ferguson, R.H. \& McConahey, W.M. (1963) The connective-tissue diseases and symptoms associated with Hashimoto's thyroiditis. New England Journal of Medicine, 268, 277.

BLAND, J.H. \& FRYMOYER, J.W. (1970) Rheumatic syndromes of myxedema. New England Journal of Medicine, 282, 1171.

Bywaters, E.G.L. (1969) Textbook of Rheumatic Diseases (Ed. by W. S. C. Copeman), 4th edn. E \& S. Livingstone Edinburgh.

Debre, R. \& Semelaigne, G. (1935) Syndrome of diffuse muscular hypertrophy in infants causing athletic appearance: its connection with congenital myxedema. American Journal of Diseases of Children, 50, 1351.

Doniach, D. (1962) Personal communication.

Fessel, W.J. (1968) Myopathy of hypothyroidism. Annals of the Rheumatic Diseases, 27, 590.

Fincham, R.W. \& CAPE, C.A. (1968) Neuropathy in myxe? dema. A study of sensory nerve conduction in the uppee extremities. Archives of Neurology, 19, 464.

GoldING, D.N. (1970) Hypothyroidism presenting wiț musculoskeletal symptoms. Annals of the Rheumati? Diseases, 29, 10.

Griffiths, P.D. (1965) Serum enzymes in diseases of the thyroid gland. Journal of Clinical Pathology, 18, 660.

Hoffman, J. (1897) Weiterer Beitrag zur Lehre von der Tetanie. Deutsche Zeitschrift fur Nervenheilkunde, 9, 278.

LeEPer, R.D., BenuA, R.S. \& BRENER, J.L. (1960) Hyperuricemia in myxedema. Journal of Clinical Endocrinology and Metabolism, 20, 1457.

NoRris, F.H. Jr \& PANNER, B.J. (1966) Hypothyroid myopathy. Clinical electromyographical and ultrastructural observations. Archives of Neurology, 14, 574.

Salick, A.I., Colachis, S.C. Jr \& Pearson, C.M. (1968) Myxedema myopathy: clinical, electrodiagnostic and pathologic findings in advanced case. Archives of Physical Medicine and Rehabilitation, 49, 230.

TALBOT, N.B. (1939) Influence of thyroid hormone on serum phosphatase. Endocrinology, 24, 230.

Weissbein, A.S., Darby, J.P. \& Lawson, J.D. (1959) An unusual bone lesion in an adult with myxedema. Archives of Internal Medicine, 104, 643.

WiLKINS, L. (1941) Epiphysical dysgenesis associated with hypothroidism. American Journal of Diseases of Children, 61, 13.

Wilkins, L. (1957) The Diagnosis and Treatment of Endocrine Disorders in Childhood and Adolescence, 2nd edn. Charles C. Thomas, Springfield, Illinois. 